\title{
Multimode multiband power amplifier with tapped transformer for efficiency enhancement in low power mode
}

\begin{abstract}
Multimode multiband connectivity has become a defacto requirement for smartphones with 3G WCDMA/4G LTE applications. In this research, a two-stage multimode multiband (MMMB) power amplifier (PA) with multiple gated transistor (MGTR) and configurable tapped transformer is designed and analysed in view of enhancing the efficiency in low power mode. The designed MMMB PA offers a $900 \mathrm{MHz}$ of operating bandwidth starting from the frequency of $1400 \mathrm{MHz}$ up to $2300 \mathrm{MHz}$, covering 16 LTE FDD bands with peak output power of $27.8 \mathrm{dBm}$ and peak PAE of $31 \%$ in the high power mode. In low power mode, the PA offers the same bandwidth with peak output power of $25.5 \mathrm{dBm}$ and PAE of $30 \%$. Use of the multiple gated transistor with tapped transformer for matching has increased the PAE in low power mode by $19 \%$ compared with that PAE in the high power mode.
\end{abstract}

Keyword: Long term evolution (LTE); Multimode multiband power amplifier; Power added efficiency (PAE); Transformer matching; WCDMA 